\title{
Decreased expression of key tumour suppressor microRNAs is associated with lymph node metastases in triple negative breast cancer
}

\author{
Kelly A Avery-Kiejda ${ }^{1,2^{*}}$, Stephen G Braye ${ }^{3}$, Andrea Mathe ${ }^{1,2}$, John F Forbes ${ }^{4,5}$ and Rodney J Scott ${ }^{1,2,3}$
}

\begin{abstract}
Background: Breast cancer is the most common malignancy that develops in women, responsible for the highest cancer-associated death rates. Triple negative breast cancers represent an important subtype that have an aggressive clinical phenotype, are associated with a higher likelihood of metastasis and are not responsive to current targeted therapies. miRNAs have emerged as an attractive candidate for molecular biomarkers and treatment targets in breast cancer, but their role in the progression of triple negative breast cancer remains largely unexplored.
\end{abstract}

Methods: This study has investigated miRNA expression profiles in 31 primary triple negative breast cancer cases and in 13 matched lymph node metastases compared with 23 matched normal breast tissues to determine miRNAs associated with the initiation of this disease subtype and those associated with its metastasis.

Results: 71 miRNAs were differentially expressed in triple negative breast cancer, the majority of which have previously been associated with breast cancer, including members of the miR-200 family and the miR-17-92 oncogenic cluster, suggesting that the majority of miRNAs involved in the initiation of triple negative breast cancer are not subtype specific. However, the repertoire of miRNAs expressed in lymph node negative and lymph node positive triple negative breast cancers were largely distinct from one another. In particular, miRNA profiles associated with lymph node negative disease tended to be up-regulated, while those associated with lymph node positive disease were down-regulated and largely overlapped with the profiles of their matched lymph node metastases. From this, 27 miRNAs were identified that are associated with metastatic capability in the triple negative breast cancer subtype.

Conclusions: These results provide novel insight into the repertoire of miRNAs that contribute to the initiation of and progression to lymph node metastasis in triple negative breast cancer and have important implications for the treatment of this breast cancer subtype.

Keywords: miRNA, Breast cancer, Triple negative, Metastases, Tumour suppressor

\section{Background}

Breast cancer is the most common malignancy that develops in women worldwide, its incidence continues to rise and it is responsible for the highest cancer-associated death rates [1]. It is an extremely heterogeneous disease, made up of a number of different subtypes. Classification of breast cancer into subtypes can be partly attributed to

\footnotetext{
* Correspondence: Kelly.Kiejda@newcastle.edu.au

${ }^{1}$ Centre for Information-Based Medicine, Hunter Medical Research Institute, New Lambton Heights, NSW 2305, Australia

${ }^{2}$ School of Biomedical Sciences and Pharmacy, Faculty of Health, University of Newcastle, Callaghan, NSW 2308, Australia

Full list of author information is available at the end of the article
}

the presence or absence of receptors for the hormones estrogen (ER), progesterone (PR) and human epidermal growth factor 2 (HER2). Triple negative breast cancer (TNBC) represents a particularly important clinical subtype, characterised by an absence of ER, PR and HER2 and which therefore lacks common targets used for antihormone therapies [2,3]. Although TNBCs comprise only a small percentage of all breast cancers diagnosed (10$24 \%$ ), they have been recently the subject of intense investigation because of their aggressive clinical behaviour. Patients who are diagnosed with TNBC are of younger age, tend to develop tumours of larger size, and have an increased likelihood of distant metastasis and death within

\section{Biomed Central}

(c) 2014 Avery-Kiejda et al.; licensee BioMed Central Ltd. This is an Open Access article distributed under the terms of the Creative Commons Attribution License (http://creativecommons.org/licenses/by/2.0), which permits unrestricted use, distribution, and reproduction in any medium, provided the original work is properly credited. 
5 years of diagnosis $[2,3]$. Thus, TNBCs represent a major clinical problem for which targeted therapies are currently not available.

microRNAs (miRNAs) are a class of small ( $\sim 22$ nucleotides) non-coding RNAs that control gene expression by targeting mRNAs and triggering either translational repression or RNA degradation [4]. miRNAs have emerged as an attractive candidate for molecular biomarkers and novel therapeutic targets in cancer because of their stability, ease of detection and their ability to act as endogenous antisense regulators of entire gene sets that regulate cancer growth [5].

Several studies have identified critical roles for miRNAs in breast cancer. Iorio and colleagues were the first to report significant deregulation in miRNA expression profiles in breast cancer when compared to normal breast tissue, where they showed that the expression of several miRNAs was associated with breast cancer subtypes and clinicopathological features including hormone receptor status, clinical stage and proliferation index [6], suggesting that miRNA expression in breast cancer may have diagnostic and prognostic value. Since then, several miRNAs have been identified that have prognostic significance, including miR-210, miR-126, miR-21 and miR-205 [7-11]. However, the clinical cohorts used to generate these miRNA profiles have been highly heterogeneous, in regards to clinicopathological variables (e.g. grade, tumour size, subtype). Moreover, because these studies have concentrated on using miRNA profiles to better classify breast cancer subtypes, there are few studies that have profiled all miRNAs within a particular breast cancer subtype and even fewer that have used matched normal tissue as controls in these analyses; and so it is plausible that miRNAs important in disease initiation and progression that are subtype specific have been missed. TNBCs are distinct from ER-, PR- positive tumours at the molecular level and this classification has clinical implications [12]. Therefore, the identification of miRNAs that control TNBC initiation and progression could identify individuals that have more aggressive disease and may also help to identify subgroups of patients that are more responsive to particular treatments within these subtypes.

The dissemination of primary cancer cells to the lymphatic system represents one of the first signs of metastatic spread. In breast cancer, the number of positive lymph nodes (LN) is known to have an inverse linear correlation with prognosis and survival [13]. This is not the case with TNBC, where it has been shown that any $\mathrm{LN}$ involvement is associated with worse disease-free and overall survival [14]. Therefore, identification of miRNAs that are differentially expressed within LN metastases in this highly aggressive breast cancer subtype may serve as a better indicator of prognosis than miRNA profiles derived from primary breast cancers.
In this study, miRNA expression profiles of 31 primary TNBCs were examined and compared to the profiles of 23 matched normal breast tissues to reveal miRNAs that were differentially expressed in TNBC and that are potentially associated with its initiation. In addition, by comparing the miRNA expression profiles of lymph node positive primary tumours and matched LN metastases, we have identified a panel of miRNAs that are associated with metastatic capability in the TNBC subtype.

\section{Methods}

\section{Study cohort and tissue sampling}

Thirty-five formalin-fixed paraffin-embedded invasive ductal carcinomas (IDCs) were obtained from the archives of the Hunter Area Pathology Service, John Hunter Hospital, Newcastle, Australia. All patients were diagnosed with grade 3 IDC between the years of 2004-2009, and were negative for ER, PR and HER2 as assessed through routine diagnostic pathology (Additional file 1: Supplementary Methods and Figure S1). Areas of tissue representing histologically normal adjacent breast tissue (NAT, where available), IDC and breast cancer metastases (in LN) were identified and confirmed by a pathologist. Micrometastases $(<2 \mathrm{~mm})$ were not used in the analysis. None of the areas of selected NAT contained tumour tissue and all were enriched for terminal duct lobular units (>2 in biopsied area). Patient information is described in Table 1. A $1.5 \mathrm{~mm}$ punch biopsy was used to punch tissue cores from the paraffin blocks using haemotoxylin and eosin stained sections of the same sample for guidance. Tumour volume in the core biopsy was $>70 \%$ of the total. This study complies with the Helsinki Declaration with ethical approval from the Hunter New England Human Research Ethics Committee (Approval number: 09/05/20/ 5.02). In accordance with the National Statement on Ethical Conduct in Research Involving Humans, a waiver of consent was granted for this study.

\section{Extraction of RNA}

Total RNA was extracted using the miRNeasy FFPE kit (Qiagen, Doncaster, VIC, Australia). RNA was quantified using the Quant-it RiboGreen RNA Assay kit (Invitrogen, Mulgrave, VIC, Australia) and purity assessed by $\mathrm{A}_{260 / \mathrm{A} 280}$ and $\mathrm{A}_{260 / 230}$ ratios ( $\left.>1.8\right)$ using the Nanodrop. The RNA integrity of selected samples was analysed using the 2100 Bioanalyser and the RNA 6000 Nano kit (Agilent Technologies, Mulgrave, VIC, Australia).

\section{miRNA arrays}

$100 \mathrm{ng}$ of total RNA was dephosphorylated and directly labelled with $\mathrm{Cy} 3$ using the miRNA Complete Labelling and Hyb Kit (Agilent Technologies). Labelled RNA was hybridised to Human miRNA microarrays (Sanger Release 14.0) according to the manufacturers' instructions (Agilent 
Table 1 Demographic data of triple negative breast cancer cases

\begin{tabular}{lll}
\hline Patient information & $\begin{array}{l}\text { Lymph node } \\
\text { negative cases (16) }\end{array}$ & $\begin{array}{l}\text { Lymph node } \\
\text { positive cases (19) }\end{array}$ \\
\hline Age (years) & $58 \pm 14$ & $54 \pm 14$ \\
Average $( \pm$ SD) & $36-84$ & $28-78$ \\
Range & $4(25 \%)$ & $9(47.3 \%)$ \\
$<50$ & $8(50 \%)$ & $7(36.8 \%)$ \\
$50-69$ & $4(25 \%)$ & $3(15.8 \%)$ \\
$>69$ & & \\
Tumour size (mm) & $32 \pm 12$ & $32 \pm 21$ \\
Average ( \pm SD) & $12-60$ & $12-100$ \\
Range & $2(12.5 \%)$ & $4(21 \%)$ \\
$<20$ & $10(62.5 \%)$ & $12(63.2 \%)$ \\
20-39 & $4(25 \%)$ & $3(15.8 \%)$ \\
$>39$ & & \\
No. of positive lymph & & $13(68.4 \%)$ \\
nodes & & $4 / 13(30.7 \%)$ \\
1-3 & 0 & $6(31.6 \%)$ \\
- Micrometastases $(<2 \mathrm{~mm})$ & 0 & \\
(No LN available) & & \\
$>3$ & 0 & $(48.9 \%)$ \\
Normal adjacent tissue & & \\
Yes & & \\
No & & \\
\hline
\end{tabular}

Technologies) and scanned on an Agilent High-resolution C scanner.

\section{miRNA array analysis}

These miRNA array results have been deposited in Gene Expression Omnibus (GEO) with Accession No. GSE38167. Data from 15,000 probe features representing 904 unique miRNAs was extracted using Agilent Feature Extraction software (v10.7.3.1) and converted to background subtracted signal intensities. The extracted data was imported into Genespring GX (Agilent Technologies) where it was $\log 2$ transformed and median normalised. Seven samples did not meet quality control measurements recommended by the manufacturer and were removed from the microarray analysis (1 IDC from a LN negative patient, 1 NAT and 3 IDCs from LN positive patients; and 2 LN metastases). Of all the probes interrogated in this analysis, those corresponding to 570 miRNA transcripts were present at a signal intensity threshold above background in at least one of the tissue samples. Unpaired $t$-tests were used to identify miRNAs with significantly altered expression ( $>2$-fold, $\mathrm{p}<0.05$ ). To correct for false positive results, a Benjamini and Hochberg False Discovery Rate (FDR) of $5.0 \%$ was used for multiple testing. Supervised hierarchical cluster analysis was performed on miRNAs that were found to be significantly different $(>2$ fold, $\mathrm{p}<0.05$, FDR $<$ 0.05). Similarity in the expression patterns between miRNAs was measured by Pearson's correlation coefficient.

Biological targets of differentially expressed miRNAs were identified by searching for the presence of conserved 8 mer and 7 mer sites within genes that match the seed region of each miRNA. Non-conserved sites were also included in this analysis. This analysis was performed using sRNA Target Base (starBase, http://starbase.sysu. edu.cn/index.php) [15] which integrates data from 21 Ago or TNRC6 CLIP-Seq sequence data sets with the target prediction programs Target Scan, Pictar and miRanda. The number of genes identified by each of these programs and those in common between each of the programs is shown in Additional file 1: Figure S2. Only those target genes that were predicted by all three target prediction programs (560 genes) were used for further pathway analysis. PANTHER [16] was used to annotate the biological pathways that predicted miRNA target genes were involved in as previously described [17]. Pathways with a pvalue $<0.05$ were considered to be significantly regulated by the miRNAs.

To determine the significance of differentially expressed miRNA families and clustered miRNAs; or to annotate the function of differentially expressed miRNAs, a freely available web-based resource, TAM (tool for annotations of miRNAs), was used [18].

\section{Semi-quantitative real-time PCR}

Total RNA (5 ng) was reverse transcribed to generate cDNA using the Taqman MicroRNA Reverse Transcription Kit and Megaplex RT Human Primer Pools Set v3.0 (Applied Biosystems, Mulgrave, VIC, Australia) according to the manufacturers' instructions. cDNA was amplified using the TaqMan PreAmp Master Mix with Megaplex Human PreAmp Primer Pools Set v3.0 (Applied Biosystems) according to the manufacturers' instructions. Realtime PCR analysis was performed in triplicate on all samples (Table 1) using TaqMan Universal PCR mix No AmpErase UNG and TaqMan MicroRNA Assays (Applied Biosystems) according to the manufacturers' instructions, with results quantified on a 7500 real-time PCR system (Applied Biosystems). The expression of the following miRNAs was analysed for array validation: hsa-let-7a (Assay ID: 377), hsa-let-7b (Assay ID: 2619), hsa-let-7c (Assay ID: 379), hsa-miR-100 (Assay ID: 437), hsa-miR101 (Assay ID: 2253), hsa-miR-126* (Assay ID:451), hsamiR-26a (Assay ID: 405), hsa-miR-26b (Assay ID: 407), hsa-miR-130a (Assay ID: 454), hsa-miR-29c (Assay ID: 587), hsa-miR-205 (Assay ID: 509), miR-210 (Assay ID: 512), RNU44 (Assay ID: 1094) and RNU49 (Assay ID: 1005). The relative miRNA expression was calculated by normalising the miRNA of interest to RNU44 $\left(2^{-\Delta \mathrm{Ct}}\right)$. Relative expression of miRNAs was also calculated using a second 
normaliser, RNU49 (data not shown). The same relative expression patterns for the miRs analysed was observed when normalised with RNU49 (examples shown in Additional file 1: Figure S3). The relative expression of RNU44 and RNU49 was not significantly different between the subgroups analysed and therefore served as an appropriate normaliser for this analysis (Additional file 1: Figure S3). All pre-amplified multiplex miRNA assays were validated against uniplex miRNA assays to verify that the multiplex reaction did not affect miRNA quantitation (Additional file 1: Supplementary Methods, Table S1 and Figure S4). hsa-miR-126* and hsa-miR-205 were outside our range of acceptable PCR efficiencies and were not used for further validations.

\section{Statistical analysis}

The normality of the data distribution was tested using a D'Agostino and Pearson Omnibus test. The values were found not to have been sampled from a Gaussian distribution and thus, non-parametric statistical tests were used to compare the real-time PCR data. A two-tailed Mann-Whitney $U$ test was used to determine if there was a statistically significant difference in the expression of miRNAs between any two subgroups. The KruskalWallis rank test followed by a Dunn's Multiple Correction test was used to determine the statistical significance of miRNA expression between multiple (>2) subgroups. Analysis of the correlation between miRNA expression and clinical parameters was performed using Spearman's correlation test. All analysis was performed using GraphPad Prism (version 5.04) software (GraphPad software Inc., La Jolla, CA, USA).

\section{Results}

\section{miRNAs differentially expressed in TNBC}

To identify miRNAs associated with TNBC, expression profiles were analysed in 35 grade 3 invasive ductal carcinomas (IDCs) compared to 24 matched normal adjacent tissue (NAT) specimens (Table 1). Seven samples did not meet quality control measurements recommended by the manufacturer and were removed from the microarray analysis (refer to Methods). Seventy-one of the miRNA transcripts were identified as being differentially expressed between IDC and NAT (Figure 1A). Supervised hierarchical clustering of these miRNAs clearly separated NAT from IDC, suggesting that the expression of these miRNAs can discriminate these two groups (Figure 1A). Five of the differentially expressed miRNAs (miR-210, miR-100, miR-130a, let-7b, let-7c) were verified by real-time PCR and were shown to be significantly different in expression between IDC and NAT in all cases (Figure 1B). In addition, the fold change in expression (IDC versus NAT) of the five
miRNAs was highly concordant $\left(R^{2}=0.9543\right)$ between microarray and real-time PCR analysis, further confirming the validity of this approach (Figure 1C).

Of the 71 significantly altered miRNA transcripts, 39 showed increased expression and 32 showed decreased expression in IDC when compared to NAT (Additional file 1: Table S2). miRNAs are known to be located in genomic clusters [19]. Several of the miRNAs that were identified as being differentially expressed are clustered within the same genomic region, and four of these genomic clusters were significantly over-represented $(\mathrm{p}<$ 0.05): the miR-17-92 cluster (miR-17, miR-18a, miR19a, miR-19b, miR-20a), the miR-106b cluster (miR-25, miR93, miR-106b), the miR-200a cluster (miR-200a, miR200b, miR-429) and the miR-106a cluster (miR-18b, miR-19b, miR-20b, miR-363) (Additional file 1: Table S3). miRNAs are commonly grouped in families based on the similarity in their seed sequence. Three miRNA families were significantly $(\mathrm{p}<0.05)$ over-represented: the miR-17 family (miR-17, miR-18a, miR-18b, miR-20a, miR-20b, miR-93, miR-106b), the miR-200 family (miR-200a, miR200b, miR-200c, miR-141, miR-429) and the miR-130 family (miR-130a, miR-130b, miR-301a) (Additional file 1: Table S3). The majority of the differentially expressed miRNAs have previously been implicated in breast cancer including miR-200a, miR-200b, miR-200c, miR-21, miR210, miR-205 and miR-10b $[7,8,10,11,20,21]$; and their regulation was concordant with previous studies. This suggests that the majority of the miRNAs identified in this study are relevant to the initiation of all breast cancers. In addition, we identified 5 miRNAs: miR-130a, miR-1280, miR-590-5p, miR-1308, miR-17*, which to the best of our knowledge, have not previously been implicated in breast cancer (Table 2). Of note, miR-130a has been shown to be associated with chemotherapy response in ovarian cancer and lung cancer cell lines, while miR-1280 has recently been demonstrated to inhibit invasion and metastasis by targeting ROCK1 when over-expressed in bladder cancer [22-24]. miR-590-5p has been reported to enhance (via the tumour suppressor PBRM1) or inhibit (via S100A10) cell growth and invasion depending on the cellular context, however, the function of miR-1308 and miR-17* has not been extensively studied $[25,26]$.

\section{miRNAs differentially expressed in LN negative IDCs compared to LN positive IDCs}

To determine miRNAs that were differentially expressed in patients with LN metastases, the primary breast cancers from $15 \mathrm{LN}$ negative patients and $16 \mathrm{LN}$ positive patients were compared. In this analysis, there were no miRNAs that were found to be significantly different. Additionally, the comparison of NAT from node negative and node positive women yielded no significant differences in miRNA profiles. 


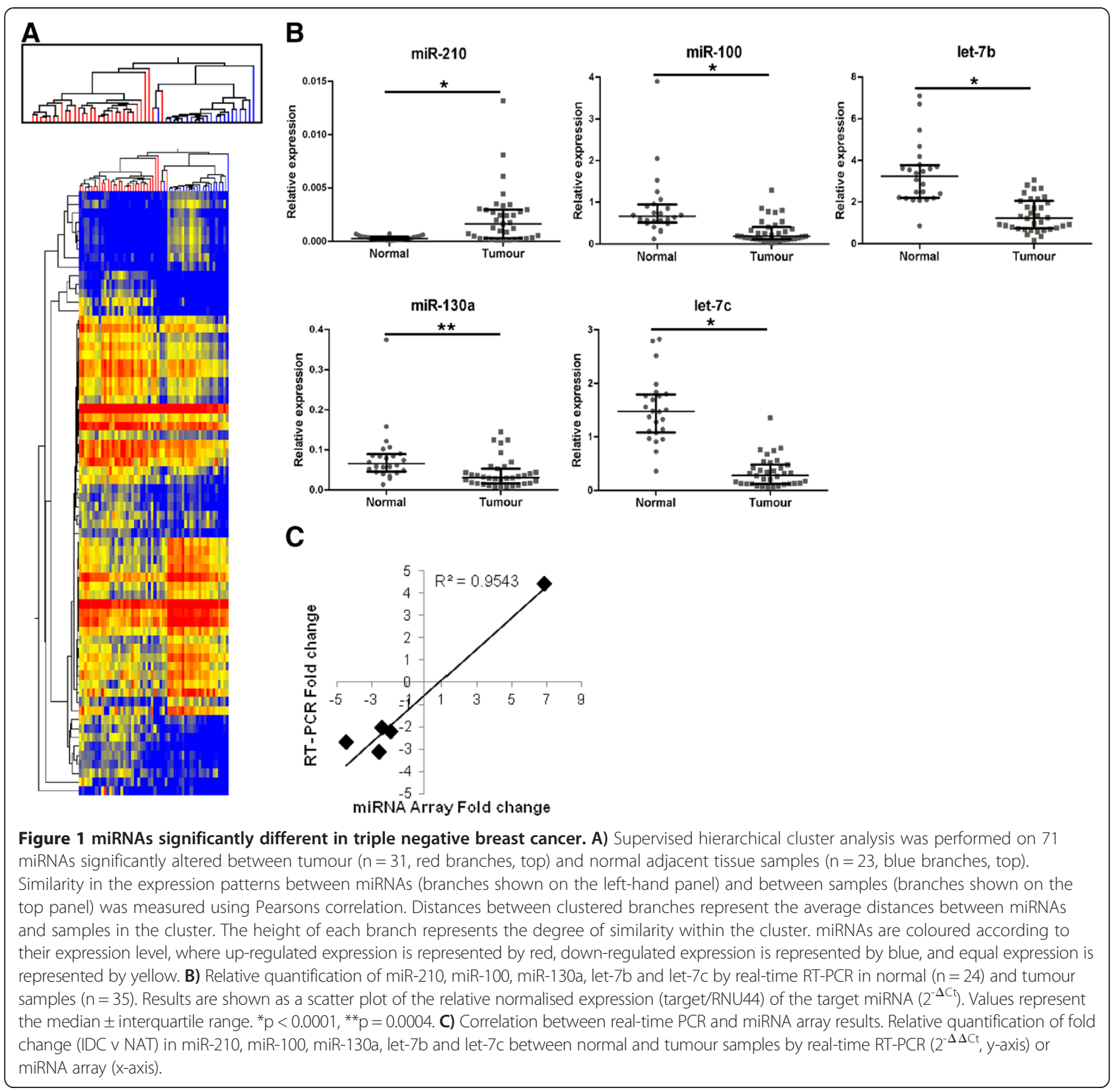

\section{miRNAs associated with LN metastasis}

In contrast to the report of Cascione et al. we undertook an additional investigation examining the relationship between lymph node positivity and miRNA expression [27]. We determined the miRNA profiles of LN positive and LN negative IDCs compared to their matched NAT. From these analyses, 37 miRNAs were found to be altered in LN negative patients, while 46 miRNAs were found to be significantly different in $\mathrm{LN}$ positive patients (Figure 2, Additional file 1: Tables S4 and S5). Supervised hierarchical clustering of these miRNAs completely separated NAT from IDC into two distinct groups in LN negative patients (Figure 2A) and was able to distinguish
Table 2 Unique miRNAs identified as being differentially expressed in triple negative breast cancer

\begin{tabular}{lll}
\hline Systematic name & Fold regulation & p-value \\
\hline hsa-miR-130a & -2.21 & 0.0160 \\
hsa-miR-1280 & 2.10 & $3.11 \mathrm{E}-04$ \\
hsa-miR-590-5p & 2.15 & 0.0131 \\
hsa-miR-1308 & 2.28 & 0.0035 \\
hsa-miR-17* & 3.03 & $3.11 \mathrm{E}-04$
\end{tabular}

Fold change in expression of 5 miRNAs found to be significantly different in extracts from 31 TNBCs compared to extracts from 23 matched normal adjacent tissue specimens ( $>2$-fold difference, $p<0.05$ and a false discovery rate $(F D R)=5.0 \%)$. 


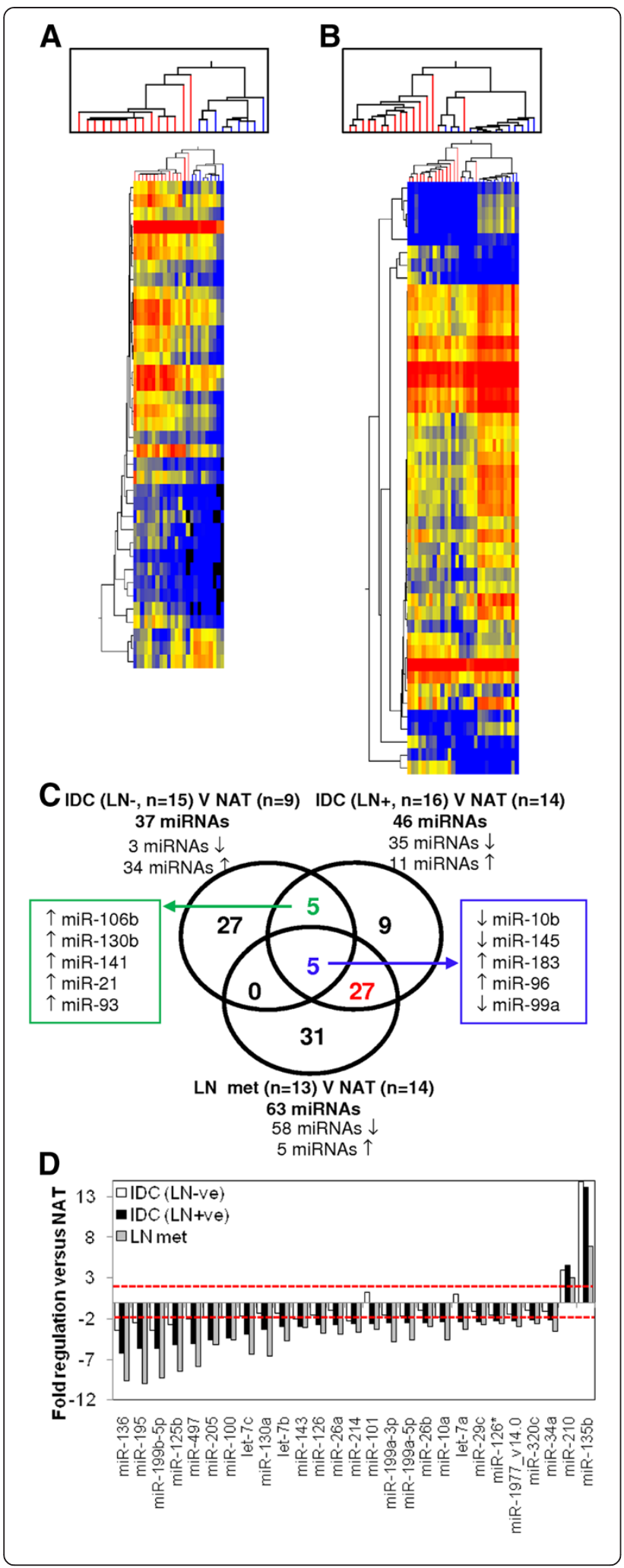

Figure 2 miRNAs significantly different in triple negative breast cancer with lymph node metastases. Supervised hierarchical cluster analysis was performed on miRNAs significantly altered between tumour (red branches, top) and normal adjacent tissue samples (blue branches, top) in $\mathbf{A}$ ) lymph node negative and B) lymph node positive patients. Similarity in the expression patterns between miRNAs (branches shown on the left-hand panel) and between samples (branches shown on the top panel) was measured using Pearsons correlation. Distances between clustered branches represent the average distances between miRNAs and samples in the cluster. The height of each branch represents the degree of similarity within the cluster. miRNAs are coloured according to their expression level, where up-regulated expression is represented by red, down-regulated expression is represented by blue, and equal expression is represented by yellow. C) Venn diagram representing the overlap between miRNAs regulated in lymph node negative patients (37 miRNAs, left-hand circle), lymph node positive patients (46 miRNAs, right-hand circle) and in the lymph node metastases of lymph node positive patients (63 miRNAs, bottom circle). D) Histogram depicting the fold regulation of the 27 miRNAs (highlighted in red in C) in tumour compared to NAT in lymph node negative tumours (white), lymph node positive tumours (black) and lymph node metastases (grey). Two-fold regulation is depicted by the red line.

the majority of LN positive IDCs (two IDCs were misclassified) from matched NAT (Figure 2B). Only 10 of the miRNAs that were identified as being differentially expressed in IDC versus NAT overlapped in LN negative and LN positive patients and the direction of their regulation (up, down) when compared to matched NAT, was concordant (Figure 2C, Additional file 1: Tables S4 and S5, miRNAs in bold). These included up-regulation of miR-21 and down-regulation of miR-10b; which have well known roles in breast cancer (Figure 2C; Additional file 1: Tables S4 and S5). Interestingly, the majority of miRNAs identified as being differentially expressed in LN negative patients were up-regulated (34/37 miRNAs), while the majority of differentially expressed miRNAs were down-regulated (35/46 miRNAs) in LN positive patients (Figure 2, Additional file 1: Tables S4 and S5). These results suggest that the repertoire of miRNAs whose expression is altered in the primary breast tumour when compared to the normal breast tissue is distinct in LN positive and negative patients.

Of note, the five unique miRNAs that were differentially regulated when all tumours were compared to all normal tissues (Table 2), were not commonly regulated when lymph node positive and negative cases were compared separately to matched normal tissue. Instead, the miRs that were positively regulated (miR-17*- 3.03, miR590-5p- 2.15, miR-1280-2.10) when all tumours were analysed became more highly up-regulated (miR-17*4.66, miR-590-5p- 3.13, miR-1280-2.40) in the comparison of lymph node negative tumours versus matched normal tissue, but were not regulated in lymph node positive tumours. In contrast, while miR-130a was down- 
regulated in the comparison of all tumour tissues $(-2.21)$, it became more strongly down-regulated in lymph node positive tumours $(-3.32)$ when they were compared to matched normal tissues, and was not regulated in lymph node negative cases. This supports our data, that there are intrinsic differences between lymph node positive and negative TNBCs: miRs are mainly down-regulated in patients with lymph node metastases, and up-regulated in patients who do not have lymph node metastases and suggests that it is these patients (i.e. lymph node positive or negative tumours) that are driving the differential regulation (negative and positive respectively) when all patients are combined.

To determine miRNAs that were altered in LN metastases, the miRNA profile of LN metastases were compared to those of NAT (9 matched and 5 unmatched) from LN positive patients. From this analysis, 63 miRNAs were found to be differentially expressed (Additional file 1 : Table S6). The majority of these miRNAs (58/63 miRNAs) were down-regulated and largely overlapped with the profile of their primary tumour (IDC, LN+; 32/63 miRNAs, Figure 2C). Many of the miRNAs overlap with those identified by Cascione et al., with 33\% (21/63) of the differentially expressed miRNAs identified by our study also showing differential regulation in normal versus LN metastases comparisons in their study of TNBC [27]. No miRNAs were differentially expressed when LN positive primary IDCs and LN metastases were compared. This suggests that the profile of miRNAs differentially expressed in primary breast cancers are maintained in LN metastases from these tumours. This is also concordant with the results of Cascione et al. who found very few differentially expressed miRNAs in this comparison [27]. In contrast, only five of the 63 miRNAs that were differentially expressed in $\mathrm{LN}$ metastases were also differentially expressed in LN negative primary IDC's (Figure 2C) when compared to NAT, suggesting that their miRNA profiles are largely distinct.

We reasoned that miRNAs that are differentially expressed in LN metastases and in the primary IDC of patients with LN metastases, that are not differentially expressed in patients who did not have LN metastases, may represent some of the earliest changes associated with metastatic progression. The overlap of the miRNA profiles is shown in Figure $2 \mathrm{C}$, where 27 miRNAs were shown to meet these criteria. These concordantly regulated miRNAs include three members of the let- 7 family (let-7a, -7b, -7c), two members of the miR-26 family (miR-26a, -26b) and three members of the miR-199 family (miR-199a-3p, -199a-5p, -199b-5p), suggesting that these miRNAs regulate similar target genes (Table 3 ). Furthermore, the list also contains miRNAs located within the same genomic cluster as one another, the let7a cluster (let-7a-3, -7b; and let-7a2, miR-100), the
miR-195 cluster (miR-195, miR-497) and the miR-199a cluster (miR-199a, miR-214), suggesting they are coordinately regulated. With the exception of miR-210 and miR-135b, all miRNAs were significantly down regulated in the primary IDC and LN metastases when compared to matched NAT, but were not significantly different in primary IDCs from LN negative patients (Figure 2D and Table 3). These results were verified by real-time PCR for nine of the miRNAs (Figure 3), where the statistical significance in the difference in expression of these miRNAs was largely confirmed, with the exception of miR101 and miR-29c, where the difference in expression of these miRNAs between normal and tumour samples in LN positive patients did not reach statistical significance.

\section{Biological functions and pathways regulated by the 27 miRNAs associated with LN metastasis}

To annotate the function of the 27 miRNAs, we performed TAM analysis [18]. Through this analysis, the majority of miRNAs were found to function as tumour suppressor miRs (13/27 miRNAs) or were involved in human embryonic stem cell regulation (11/27 miRNAs) and these functions were significantly over-represented. A number of miRNAs in this list are also known to have functions in cell death and/or the cell cycle and these functions were also significantly over-represented (Additional file 1: Table S7).

We next used starBase and PANTHER to predict the genes and signalling pathways regulated by these miRNAs. Ten pathways were predicted to be significantly regulated by these miRNAs and many of these have already been reported to be dysregulated in TNBCs including the p53, EGFR1, Wnt and the TGF $\beta$ signalling pathways (Table 4).

\section{Correlation between miRNA expression and clinical parameters}

To determine if the expression of miRNAs verified in this study by real-time PCR were independently related to age at diagnosis, tumour size or the percentage of positive LNs, we tested their association using Spearman's Rank correlation (Table 5). None of the miRNAs tested were independently associated with age at diagnosis or tumour size. However, the expression of miR-101 was negatively correlated with the percentage of LNs positive while the negative correlation of let-7b and miR-29c with the percentage of LNs positive approached significance (Table 5).

\section{Discussion}

This study has investigated miRNA profiles in TNBC cases to determine miRNAs whose expression is associated with the initiation of this breast cancer subtype and those associated with its metastasis to the LN. We have 
Table 3 miRNAs differentially expressed in triple negative breast cancer patients with lymph node metastases

\begin{tabular}{|c|c|c|c|c|c|c|}
\hline \multirow[b]{3}{*}{ Systematic name } & \multicolumn{4}{|c|}{ Lymph node positive cases } & \multirow{2}{*}{\multicolumn{2}{|c|}{$\begin{array}{l}\text { Lymph node negative cases } \\
\text { IDC v NAT }\end{array}$}} \\
\hline & \multicolumn{2}{|l|}{ IDC v NAT } & \multicolumn{2}{|l|}{ LN met v NAT } & & \\
\hline & Fold change & p-value & Fold change & p-value & Fold change & p-value \\
\hline hsa-let-7a & -2.36 & 0.00419 & -3.33 & 0.00042 & 1.05 & ns \\
\hline hsa-let-7b & -2.99 & 0.00011 & -4.67 & 0.00007 & -1.30 & ns \\
\hline hsa-let-7c & -3.84 & 0.00006 & -6.34 & 0.00001 & -1.72 & ns \\
\hline hsa-miR-100 & -4.37 & 0.00176 & -4.57 & 0.00331 & -1.84 & ns \\
\hline hsa-miR-101 & -2.61 & 0.01750 & -3.30 & 0.02278 & 1.22 & ns \\
\hline hsa-miR-10a & -2.37 & 0.02228 & -4.64 & 0.00496 & -1.78 & ns \\
\hline hsa-miR-125b & -5.18 & 0.00010 & -8.41 & 0.00001 & -2.69 & ns \\
\hline hsa-miR-126 & -2.72 & 0.00451 & -3.76 & 0.00451 & -1.50 & ns \\
\hline hsa-miR-126* & -2.31 & 0.04409 & -2.63 & 0.02725 & -1.56 & ns \\
\hline hsa-miR-130a & -3.32 & 0.01446 & -6.61 & 0.00430 & -1.32 & ns \\
\hline hsa-miR-135b & 14.14 & 0.00012 & 6.92 & 0.02725 & 14.87 & ns \\
\hline hsa-miR-136 & -6.18 & 0.00011 & -9.58 & 0.00223 & -3.42 & ns \\
\hline hsa-miR-143 & -2.99 & 0.01750 & -3.04 & 0.03718 & -2.02 & ns \\
\hline hsa-miR-195 & -5.70 & 0.00006 & -10.02 & 0.00029 & -2.50 & ns \\
\hline hsa-miR-1977_v14.0 & -2.28 & 0.00377 & -2.91 & 0.00069 & -1.49 & ns \\
\hline hsa-miR-199a-3p & -2.50 & 0.01750 & -4.84 & 0.00451 & -1.55 & ns \\
\hline hsa-miR-199a-5p & -2.46 & 0.04409 & -4.64 & 0.00869 & -1.66 & ns \\
\hline hsa-miR-199b-5p & -5.66 & 0.00011 & -9.29 & 0.00021 & -3.40 & ns \\
\hline hsa-miR-205 & -4.65 & 0.02890 & -5.18 & 0.01648 & -1.82 & ns \\
\hline hsa-miR-210 & 4.56 & 0.00862 & 2.98 & 0.02389 & 4.01 & ns \\
\hline hsa-miR-214 & -2.64 & 0.01360 & -3.64 & 0.02023 & -2.28 & ns \\
\hline hsa-miR-26a & -2.67 & 0.01428 & -3.84 & 0.00247 & -1.00 & ns \\
\hline hsa-miR-26b & -2.43 & 0.01681 & -3.01 & 0.01308 & -1.01 & ns \\
\hline hsa-miR-29c & -2.33 & 0.04343 & -2.69 & 0.03676 & -1.12 & ns \\
\hline hsa-miR-320c & -2.15 & 0.02461 & -2.65 & 0.02023 & -1.02 & ns \\
\hline hsa-miR-34a & -2.12 & 0.02087 & -3.59 & 0.00825 & -1.11 & ns \\
\hline hsa-miR-497 & -5.11 & 0.00006 & -7.91 & 0.00029 & -2.06 & ns \\
\hline
\end{tabular}

Fold change in expression of 27 miRNAs found to be significantly different in extracts from 16 TNBC cases (lymph node positive) and their corresponding lymph node metastases ( 13 cases) when compared to their matched normal adjacent tissue specimens ( 14 cases) ( $>2$-fold difference, $p<0.05$ and a false discovery rate $(F D R)=5.0 \%$ ). Expression of these miRNAs in lymph node negative cases (IDC $n=15$, NAT $n=9$ ) is shown for comparison. ns $=$ not significant.

identified a panel of 27 miRNAs that are associated with breast cancer progression in TNBC.

\section{miRNAs differentially expressed in TNBC}

By comparing the miRNA profiles of primary breast tumours to matched normal adjacent breast tissue, 71 miRNAs were identified as being differentially expressed. The majority of the miRNAs identified in this analysis have previously been implicated in breast cancer, suggesting that these miRNAs have a general function in the tumourigenic process in all breast cancers and that they are not specific to TNBC. This is perhaps not surprising, given the weak separation of the TNBC subtype by hierarchical clustering in miRNA expression profiles when compared to other breast tumour subtypes [28].
The results of this study are highly concordant with that of Cascione et al., with 70\% (50/71) of the differentially expressed miRNAs identified by our study also showing differential regulation in normal versus tumour comparisons in their study of TNBC [27]. Differentially expressed miRNAs included members of the miR-17-92 oncogenic cluster, the let-7 family and the miR-200 family which are known to be dysregulated in several solid tumours, including breast cancer [29,30]. Of particular note, the miR-17-92 cluster was recently shown to be up-regulated in TNBC and the basal subtype [10,31] while miR-145 and miR-199b-5p were strongly repressed [10] consistent with our results. miR-210 and miR-21, well known oncomiRs $[7,11]$ were found to be overexpressed in our study. We also observed that miRNAs 


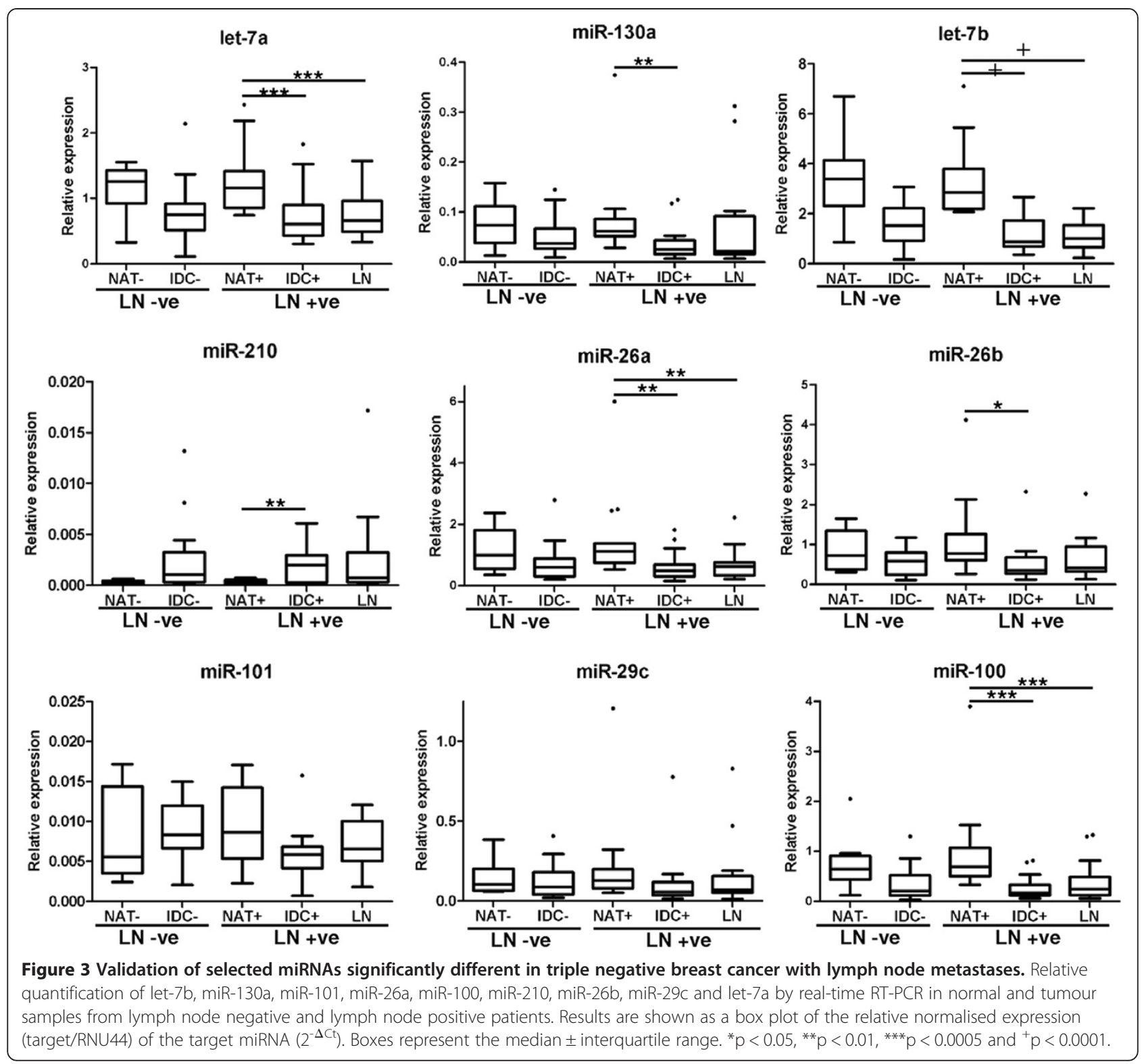

known to be bona fide regulators of ER $\alpha$ (miR-18a, miR18b) were over-expressed [32]; while those that have been previously reported to be differentially expressed between ER-positive and -negative breast cancers (including let-7b, miR-200a, miR-21, miR-25, miR-106b) were significantly altered in this study [32].

The immunohistochemical and molecular profiles of TNBCs are similar to that of hereditary breast cancers that have mutations in the BRCA1 gene [2]. In this regard, several miRNAs identified in this study are known to participate in the BRCA1 signalling axis including miR-146a, miR-155 and miR-335 [33-35]. Deregulation of these miRNAs in TNBC may contribute to altered BRCA1 signalling and could partly explain the similarities of these tumours with those in which BRCA1 function is lost.
miRNAs involved in LN positive TNBC

A significant and important finding from this study is that the miRNA profiles of $\mathrm{LN}$ positive primary breast cancers were strikingly distinct from that of LN negative patients. In particular, there was an overall up-regulation of miRNAs in LN negative patients and a dampening of miRNA expression in LN positive patients, suggesting that oncogenic miRNAs are associated with the development of LN negative TNBC and in contrast, that deregulated expression of tumour suppressor miRs is involved in LN positive disease. Two enzymes, Drosha and Dicer, are pivotal in the processing of pri-miRNA into mature double stranded miRNA fragments [4]. Interestingly, in breast cancer, reduced expression of Dicer has been associated with shorter metastasis-free survival and with 
Table 4 Predicted signalling pathways regulated by 27 differentially expressed miRNAs in TNBCs with LN metastases

\begin{tabular}{|c|c|c|c|c|c|}
\hline Pathway & $\begin{array}{l}\text { Homo sapiens } \\
\text { reference genome } \\
\text { (No. of genes) }\end{array}$ & $\begin{array}{l}\text { miRNA target genes } \\
\text { (No. of genes) }\end{array}$ & Expected & $\begin{array}{l}\text { Over/under } \\
\text { represented (+/-) }\end{array}$ & $p$ value \\
\hline Wnt signaling pathway & 318 & 16 & 5.69 & + & 0.000248 \\
\hline TGF-beta signaling pathway & 149 & 10 & 2.66 & + & 0.000423 \\
\hline Cell cycle & 22 & 4 & 0.39 & + & 0.00072 \\
\hline p53 pathway & 114 & 7 & 2.04 & + & 0.00487 \\
\hline p53 pathway by glucose deprivation & 25 & 3 & 0.45 & + & 0.0106 \\
\hline p53 pathway feedback loops & 52 & 4 & 0.93 & + & 0.0148 \\
\hline PI3 kinase pathway & 117 & 6 & 2.09 & + & 0.0198 \\
\hline $\begin{array}{l}\text { Heterotrimeric G-protein signaling pathway-Gi alpha and Gs } \\
\text { alpha mediated pathway }\end{array}$ & 164 & 7 & 2.93 & + & 0.0296 \\
\hline EGF receptor signaling pathway & 130 & 6 & 2.32 & + & 0.0308 \\
\hline De novo pyrmidine ribonucleotides biosythesis & 18 & 2 & 0.32 & + & 0.0418 \\
\hline
\end{tabular}

the TNBC subtype, where it is observed in $60-78 \%$ of patients [36-38]. The correlation of Dicer expression with LN metastases in TNBC patients was not examined in these studies [36,38]. It could be hypothesised that the overall down-regulation of miRNAs observed in LN positive TNBCs in the current study is a result of reduced Dicer expression, but this remains to be determined.

\section{miRNAs as markers for metastasis}

In this study, we were not seeking to identify miRNAs that were differentially expressed in the transition of breast cancer progression i.e. primary breast cancer to metastasis or from ductal carcinoma in situ (DCIS) to IDC, as previous studies have done $[10,27,28,39]$. In contrast to other studies, our analysis yielded no discriminatory miRNAs when matched primary breast cancer and LN metastases were compared suggesting that they are remarkably similar in their miRNA profiles and supporting the validity of our approach. The premise of the current study was to determine altered miRNA expression patterns in primary breast cancers that were also present in LN metastases. We reasoned that deregulated expression of key miRNAs that promote a highly invasive phenotype would be an early event in breast cancer progression and that these changes would be present in both the primary and metastatic lesion. Using this approach, we identified a panel of 27 miRNAs that are associated with metastasis to the LN. Given that the majority of these miRNAs are down-regulated, their value as prognostic markers remains to be determined and is the subject of ongoing investigations. However, 17/27 (63\%) miRNAs are either known to be tumour suppressor miRs and/or have been shown to play a role in human embryonic stem cell regulation (Additional file 1: Table S7), supporting that deregulated expression of this panel of miRNAs is likely to result in altered differentiation states and increased invasiveness. In this regard, let7 family members, miR-34a and miR-205 are markedly

Table 5 Correlation between miRNA expression levels and clinical variables in triple negative breast cancers

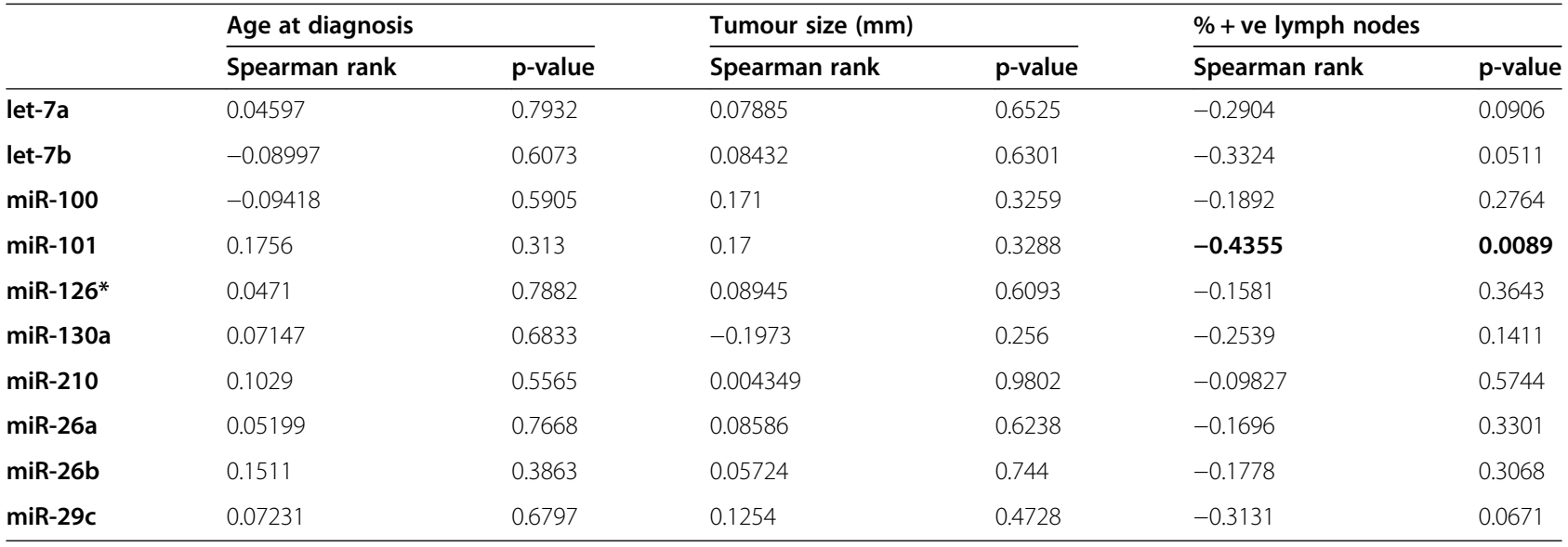

Values represent Spearman's rank correlation coefficient and significant correlations $(p<0.05)$ are shown in bold. 
reduced in breast tumour initiating cells and are involved in epithelial to mesenchymal transition [21,40,41]. In addition, several of the miRNAs in this list have already been shown to have prognostic value in breast cancer including miR-210, miR-126, miR-26a, miR-125b, miR-205 and miR-214 [7-10,42-44]. Furthermore, our results are in agreement with Cascione et al., who found that both miR$125 \mathrm{~b}$ and miR-497 were down-regulated and related to survival in TNBC [27].

Within our panel of metastasis-related miRNAs are a number of miRNAs whose functional role in breast cancer progression has not previously been described. These include miR-320c, miR-29c, miR-130a and miR195 among others. There are no reports on miR-320c function to date and thus its possible role in promoting LN metastasis in TNBC remains unknown. miR-29c has previously been reported to be up-regulated in a small cohort of breast cancer cases [11], however, we found miR-29c to be down-regulated and associated with LN metastasis. In agreement with our study, miR-29c is down-regulated in chronic lymphocytic leukaemia and a range of solid tumours including colorectal cancer where it has been reported to decrease with cancer progression and is a predictor of survival and early recurrence $[45,46]$. miR-130a is down-regulated in a panel of ovarian cancer and lung cancer cell lines that are resistant to chemotherapies [23,24]. Moreover, it has recently been shown to be decreased in prostate cancer and its overexpression in prostate cancer cell lines causes the repression of key oncogenic pathways such as the MAPK pathway [47]. miR-195 was markedly down-regulated in our study. Its re-expression in breast cancer cell lines has recently been reported to reduce cellular proliferation and invasion, suggesting that it plays a key role in breast cancer progression [48].

A recent study by Buffa et al., described three miRNAs (miR-342, miR-27b, miR-150) that were prognostic for relapse-free survival in TNBC [49]. In our hands, these results were not replicated for $\mathrm{LN}$ metastasis and moreover, these miRNAs were not differentially expressed when breast tumour was compared to normal tissue. In addition, miR-10b has been shown to be strongly expressed in metastatic breast cancer cells, where it regulates invasion and migration [50]. However, we observed miR-10b to be strongly down-regulated in lymph-node positive and negative primary breast cancers and in LN metastases, suggesting it does not contribute to breast cancer invasiveness, and this is in strong agreement with several recent studies $[6,10,20]$.

We used starBASE to predict signalling pathways regulated by the 27 miRNAs associated with LN metastasis. Interestingly, the pathways identified by this analysis have been widely reported to be deregulated in TNBC. In particular Wnt/ $\beta$-Catenin, EGFR1 and the TGF $\beta$ receptor signalling axis are all known to be highly expressed in a large proportion of cases within the TNBC subtype and have been proposed as alternative treatment targets for TNBC $[2,3,51]$. A number of chemotherapeutic agents which target these pathways are currently under investigation in patients with TNBC, in both the neoadjuvant and metastatic setting [52]. The findings from our study- decreased expression of key miRNAs which target these pathways- provides a biological mechanism for up-regulation of these pathways in this breast cancer subtype. Additionally, TP53, PIK3CA and EGFR are among the four most commonly mutated genes associated with aberrant expression of interacting genes in TNBC [53], exemplifying their importance in this subtype. The predicted regulation of these pathways by miRNAs, as found in our study, reveals another mechanism for disruption of these pathways in TNBC.

\section{Study design}

A disadvantage of our study design is that samples were acquired by punch biopsy, rather than isolating individual normal breast and tumour epithelial cells by laser capture microdissection. We recognise that tissue heterogeneity and contamination by other non-neoplastic cell types including adipocytes, stroma and lymphocytes was unavoidable and that it may have contributed to the miRNA profiles generated. Our approach of selecting miRNAs that were only differentially expressed in primary tumours and matched LN metastases and that were not altered in LN negative primary tumours would have culled a large proportion of those miRNAs that are specifically altered in inflammatory responses. Moreover, those miRNAs that were differentially expressed simply because of differences in cell type composition between normal tissue (higher content of adipocytes and stroma) when compared to tumour tissue (higher content of epithelium) would have shown overlapping miRNA profiles when both $\mathrm{LN}$ negative and $\mathrm{LN}$ positive primary tumours were compared to matched normal tissues (10 miRNAs, Figure 2C) and were not included in our list of miRNAs related to metastasis (Table 3 ). Therefore, because of this careful process of elimination, we consider the miRNAs identified in this study (Table 3) are strongly representative of the invasive capacity of the primary tumour.

Given that the sample size used in this study is relatively small, conclusions cannot be drawn about the relationship of these miRNAs to prognosis or their specificity to the TNBC subtype. However, the tumours are homogenous with regards to size, hormone receptor status and histological grade. Furthermore, the size of this cohort $(n=35)$ is in line with several recent studies investigating the prognostic value of miRNAs in TNBC (Buffa et al., $\mathrm{n}=37$; Farazi et al., $\mathrm{n}=48$; Radojocic et al., 
$\mathrm{n}=49)[20,28,49]$ and represents the only study to date that has analysed the full repertoire of miRNAs in lymph node positive and negative TNBC samples separately compared to matched normal adjacent tissue. The uniqueness of our study design was highly advantageous for delineating critical differences in miRNA profiles between LN positive and negative primary tumours, which would have been missed if: 1) matched normal adjacent tissue was not used and 2) only primary tumours from LN positive versus LN negative patients were compared to one another. In a recent study, Farazi and colleagues showed that there was no difference in miRNA profiles of primary breast tumours between TNBC patients who relapsed when compared to those who did not [28]. Indeed, we also found that no miRNAs were differentially expressed when LN negative versus positive primary tumours were directly compared using our statistical cutoffs. Further to this, in our study no miRNAs were found to be differentially expressed when normal tissue from LN positive patients was compared to that of LN negative patients. As such, the distinct miRNA profiles generated when LN negative and LN positive primary tumours were each separately compared to matched normal adjacent tissues are likely to reflect small, but critical changes in the regulation of miRNAs that are essential for the development of each of these respective phenotypes. Given that any LN involvement (regardless of number) is associated with worse disease-free and overall survival in TNBC [14], identification of molecular determinants such as miRNAs, that contribute to this highly invasive phenotype is urgently needed.

\section{Conclusions}

This study has provided novel insight into the repertoire of miRNAs that contribute to the initiation of and progression to LN metastasis in TNBC. These miRNAs may serve as markers for metastasis or treatment targets in the future.

\section{Additional file}

Additional file 1: Further characterisation of miRNAs in triple negative breast cancer. Contains Supplementary methods, Tables S1-S7 and Figures S1-S4.

\footnotetext{
Abbreviations

miRNA: Micro ribonucleic acid; ER: Estrogen receptor; PR: Progesterone receptor; HER2: Human epidermal growth factor receptor 2; TNBC: Triple negative breast cancer; mRNA: Messenger ribonucleic acid; LN: Lymph node; IDC: Invasive ductal carcinoma; NAT: Normal adjacent tissue;

cDNA: Complementary deoxyribonucleic acid.
}

Competing interests

The authors declare they have no competing interests.

\section{Authors' contributions}

KAK: study concept and design, carried out experiments, analysis and interpretation of data, drafting of the manuscript. SGB: patient collection, material support, manuscript revision. AM: analysis and interpretation of data. JFF: study design, obtained funding, critical revision of the manuscript for important intellectual content. RJS: study design, obtained funding, critical revision of the manuscript for important intellectual content. All authors read and approved the final manuscript.

\section{Acknowledgements}

The authors would like to thank Dr. Ricardo Vilain for pathological review of all tumour and normal tissue specimens used in this analysis and Ms. Tina Hope for assistance with archival specimens. This work was supported by funding from the National Breast Cancer Foundation and the Hunter Medical Research Institute. Dr. Avery-Kiejda is supported by a Hunter Translational Cancer Research Unit Fellowship from the Cancer Institute NSW.

\section{Author details}

${ }^{1}$ Centre for Information-Based Medicine, Hunter Medical Research Institute, New Lambton Heights, NSW 2305, Australia. ${ }^{2}$ School of Biomedical Sciences and Pharmacy, Faculty of Health, University of Newcastle, Callaghan, NSW 2308, Australia. ${ }^{3}$ Hunter Area Pathology Service, John Hunter Hospital, New Lambton Heights, NSW 2305, Australia. ${ }^{4}$ Department of Surgical Oncology, Calvary Mater Newcastle Hospital, Australian New Zealand Breast Cancer Trials Group, Waratah, NSW 2298, Australia. ${ }^{5}$ School of Medicine and Public Health, Faculty of Health, University of Newcastle, Callaghan, NSW 2308, Australia.

Received: 5 August 2013 Accepted: 29 January 2014

Published: 31 January 2014

\section{References}

1. Kamangar F, Dores GM, Anderson WF: Patterns of cancer incidence, mortality, and prevalence across five continents: defining priorities to reduce cancer disparities in different geographic regions of the world. J Clin Oncol 2006, 24(14):2137-2150.

2. Podo F, Buydens LM, Degani H, Hilhorst R, Klipp E, Gribbestad IS, Van Huffel S, van Laarhoven HW, Luts J, Monleon D, Postma GJ, Schneiderhan-Marra N, Santoro F, Wouters H, Russnes HG, Sorlie T, Tagliabue E, Borresen-Dale AL: Triple-negative breast cancer: present challenges and new perspectives. Mol Oncol 2010, 4(3):209-229.

3. Carey L, Winer E, Viale G, Cameron D, Gianni L: Triple-negative breast cancer: disease entity or title of convenience? Nat Rev Clin Oncol 2010, 7(12):683-692.

4. Jackson RJ, Standart N: How do microRNAs regulate gene expression? Sci STKE 2007, 2007(367):re1.

5. Andorfer CA, Necela BM, Thompson EA, Perez EA: MicroRNA signatures: clinical biomarkers for the diagnosis and treatment of breast cancer. Trends Mol Med 2011, 17(6):313-319.

6. Iorio MV, Ferracin M, Liu CG, Veronese A, Spizzo R, Sabbioni S, Magri E, Pedriali M, Fabbri M, Campiglio M, Menard S, Palazzo JP, Rosenberg A, Musiani P, Volinia S, Nenci I, Calin GA, Querzoli P, Negrini M, Croce CM: MicroRNA gene expression deregulation in human breast cancer. Cancer Res 2005, 65(16):7065-7070.

7. Foekens JA, Sieuwerts AM, Smid M, Look MP, de Weerd V, Boersma AW, Klijn JG, Wiemer EA, Martens JW: Four miRNAs associated with aggressiveness of lymph node-negative, estrogen receptor-positive human breast cancer. Proc Natl Acad Sci USA 2008, 105(35):13021-13026.

8. Le Quesne JL, Jones J, Warren J, Dawson SJ, Ali R, Bardwell H, Blows F Pharoah P, Caldas C: Biological and prognostic associations of miR-205 and let-7b in breast cancer revealed by in situ hybridisation analysis of micro-RNA expression in arrays of archival tumour tissue. J Pathol 2012, 227(3):306-314.

9. Rothe F, Ignatiadis M, Chaboteaux C, Haibe-Kains B, Kheddoumi N, Majjaj S, Badran B, Fayyad-Kazan H, Desmedt C, Harris AL, Piccart M, Sotiriou C: Global microRNA expression profiling identifies MiR-210 associated with tumor proliferation, invasion and poor clinical outcome in breast cancer. PLoS One 2011, 6(6):e20980.

10. Volinia S, Galasso M, Sana ME, Wise TF, Palatini J, Huebner K, Croce CM: Breast cancer signatures for invasiveness and prognosis defined by deep sequencing of microRNA. Proc Natl Acad Sci USA 2012, 109(8):3024-3029. 
11. Yan LX, Huang XF, Shao Q, Huang MY, Deng L, Wu QL, Zeng YX, Shao JY: MicroRNA miR-21 overexpression in human breast cancer is associated with advanced clinical stage, lymph node metastasis and patient poor prognosis. RNA 2008, 14(11):2348-2360.

12. Sorlie T, Perou CM, Tibshirani R, Aas T, Geisler S, Johnsen H, Hastie T, Eisen $M B$, van de Rijn M, Jeffrey SS, Thorsen T, Quist H, Matese JC, Brown PO, Botstein D, Eystein Lonning P, Borresen-Dale AL: Gene expression patterns of breast carcinomas distinguish tumor subclasses with clinical implications. Proc Nat Acad Sci USA 2001, 98(19):10869-10874.

13. Carter $\mathrm{CL}$, Allen $\mathrm{C}$, Henson DE: Relation of tumor size, lymph node status, and survival in 24,740 breast cancer cases. Cancer 1989, 63(1):181-187.

14. Hernandez-Aya LF, Chavez-Macgregor M, Lei X, Meric-Bernstam F, Buchholz TA, Hsu L, Sahin AA, Do KA, Valero V, Hortobagyi GN, Gonzalez-Angulo AM: Nodal status and clinical outcomes in a large cohort of patients with triple-negative breast cancer. J Clin Oncol 2011, 29(19):2628-2634.

15. Yang JH, Li JH, Shao P, Zhou H, Chen YQ, Qu LH: starBase: a database for exploring microRNA-mRNA interaction maps from Argonaute CLIP-Seq and Degradome-Seq data. Nucleic Acids Res 2011, 39(Database issue):D202-D209.

16. PANTHER Classification system. http://www.pantherdb.org.

17. Avery-Kiejda KA, Bowden NA, Croft AJ, Scurr LL, Kairupan CF, Ashton KA, Talseth-Palmer BA, Rizos H, Zhang XD, Scott RJ, Hersey P: P53 in human melanoma fails to regulate target genes associated with apoptosis and the cell cycle and may contribute to proliferation. BMC Cancer 2011, 11(1):203.

18. Lu M, Shi B, Wang J, Cao Q, Cui Q: TAM: a method for enrichment and depletion analysis of a microRNA category in a list of microRNAs. BMC Bioinformatics 2010, 11:419.

19. Altuvia $Y$, Landgraf $P$, Lithwick $G$, Elefant $N$, Pfeffer $S$, Aravin A, Brownstein MJ, Tuschl T, Margalit $\mathrm{H}$ : Clustering and conservation patterns of human microRNAs. Nucleic Acids Res 2005, 33(8):2697-2706.

20. Radojicic J, Zaravinos A, Vrekoussis T, Kafousi M, Spandidos DA, Stathopoulos EN: MicroRNA expression analysis in triple-negative (ER, PR and Her2/neu) breast cancer. Cell Cycle 2011, 10(3):507-517.

21. Gregory PA, Bert AG, Paterson EL, Barry SC, Tsykin A, Farshid G, Vadas MA, Khew-Goodall Y, Goodall GJ: The miR-200 family and miR-205 regulate epithelial to mesenchymal transition by targeting ZEB1 and SIP1. Nat Cell Biol 2008, 10(5):593-601.

22. Majid S, Dar AA, Saini S, Shahryari V, Arora S, Zaman MS, Chang I, Yamamura S, Chiyomaru T, Fukuhara S, Tanaka Y, Deng G, Tabatabai ZL, Dahiya R: MicroRNA-1280 inhibits invasion and metastasis by targeting ROCK1 in bladder cancer. PLoS One 2012, 7(10):e46743.

23. Acunzo M, Visone R, Romano G, Veronese A, Lovat F, Palmieri D, Bottoni A, Garofalo M, Gasparini P, Condorelli G, Chiariello M, Croce CM: miR-130a targets MET and induces TRAIL-sensitivity in NSCLC by downregulating miR-221 and 222. Oncogene 2012, 31(5):634-642.

24. Sorrentino A, Liu CG, Addario A, Peschle C, Scambia G, Ferlini C: Role of microRNAs in drug-resistant ovarian cancer cells. Gynecol Oncol 2008, 111(3):478-486.

25. Shan X, Miao Y, Fan R, Qian H, Chen P, Liu H, Yan X, Li J, Zhou F: MiR-590-5P Inhibits Growth of HepG2 Cells via Decrease of S100A10 Expression and Inhibition of the Wnt Pathway. Int J Mol Sci 2013, 14(4):8556-8569.

26. Xiao X, Tang C, Xiao S, Fu C, Yu P: Enhancement of proliferation and invasion by MicroRNA-590-5p via targeting PBRM1 in clear cell renal carcinoma cells. Oncol Res 2013, 20(11):537-544.

27. Cascione L, Gasparini P, Lovat F, Carasi S, Pulvirenti A, Ferro A, Alder $\mathrm{H}, \mathrm{He}$ $G$, Vecchione A, Croce CM, Shapiro CL, Huebner K: Integrated microRNA and mRNA signatures associated with survival in triple negative breast cancer. PLoS One 2013, 8(2):e55910.

28. Farazi TA, Horlings HM, Ten Hoeve JJ, Mihailovic A, Halfwerk H, Morozov P, Brown M, Hafner M, Reyal F, van Kouwenhove M, Kreike B, Sie D, Hovestadt V, Wessels LF, van de Vijver MJ, Tuschl T: MicroRNA sequence and expression analysis in breast tumors by deep sequencing. Cancer Res 2011, 71(13):4443-4453.

29. Blenkiron C, Goldstein LD, Thorne NP, Spiteri I, Chin SF, Dunning MJ, Barbosa-Morais NL, Teschendorff AE, Green AR, Ellis IO, Tavare S, Caldas C, Miska EA: MicroRNA expression profiling of human breast cancer identifies new markers of tumor subtype. Genome Bio/ 2007, 8(10):R214.

30. Volinia S, Calin GA, Liu CG, Ambs S, Cimmino A, Petrocca F, Visone R, lorio M, Roldo C, Ferracin M, Prueitt RL, Yanaihara N, Lanza G, Scarpa A, Vecchione A, Negrini M, Harris CC, Croce CM: A microRNA expression signature of human solid tumors defines cancer gene targets. Proc Nat Acad Sci USA 2006, 103(7):2257-2261.
31. Enerly E, Steinfeld I, Kleivi K, Leivonen SK, Aure MR, Russnes HG, Ronneberg JA, Johnsen $H$, Navon R, Rodland E, Makela R, Naume B, Perala M, Kallioniemi O, Kristensen VN, Yakhini Z, Borresen-Dale AL: miRNA-mRNA integrated analysis reveals roles for miRNAs in primary breast tumors. PLoS One 2011, 6(2):e16915.

32. Klinge CM: miRNAs and estrogen action. Trends Endocrinol Metab 2012, 23(5):223-233.

33. Garcia Al, Buisson M, Bertrand P, Rimokh R, Rouleau E, Lopez BS, Lidereau R, Mikaelian I, Mazoyer S: Down-regulation of BRCA1 expression by miR-146a and miR-146b-5p in triple negative sporadic breast cancers. EMBO Mol Med 2011, 3(5):279-290.

34. Chang S, Wang RH, Akagi K, Kim KA, Martin BK, Cavallone L, Haines DC, Basik M, Mai P, Poggi E, Isaacs C, Looi LM, Mun KS, Greene MH, Byers SW, Teo SH, Deng CX, Sharan SK: Tumor suppressor BRCA1 epigenetically controls oncogenic microRNA-155. Nat Med 2011, 17(10):1275-1282.

35. Heyn $H$, Engelmann M, Schreek S, Ahrens P, Lehmann U, Kreipe $H$, Schlegelberger B, Beger C: MicroRNA miR-335 is crucial for the BRCA1 regulatory cascade in breast cancer development. Int J Cancer 2011, 129(12):2797-2806.

36. Dedes KJ, Natrajan R, Lambros MB, Geyer FC, Lopez-Garcia MA, Savage K, Jones RL, Reis-Filho JS: Down-regulation of the miRNA master regulators Drosha and Dicer is associated with specific subgroups of breast cancer. Eur J Cancer 2011, 47(1):138-150.

37. Grelier G, Voirin N, Ay AS, Cox DG, Chabaud S, Treilleux I, Leon-Goddard S, Rimokh R, Mikaelian I, Venoux C, Puisieux A, Lasset C, Moyret-Lalle C: Prognostic value of Dicer expression in human breast cancers and association with the mesenchymal phenotype. Br J Cancer 2009, 101(4):673-683.

38. Passon N, Gerometta A, Puppin C, Lavarone E, Puglisi F, Tell G, Di Loreto C, Damante G: Expression of Dicer and Drosha in triple-negative breast cancer. J Clin Pathol 2012, 65(4):320-326.

39. Baffa R, Fassan M, Volinia S, O'Hara B, Liu CG, Palazzo JP, Gardiman M, Rugge M, Gomella LG, Croce CM, Rosenberg A: MicroRNA expression profiling of human metastatic cancers identifies cancer gene targets. J Pathol 2009, 219(2):214-221.

40. Kim NH, Kim HS, Li XY, Lee I, Choi HS, Kang SE, Cha SY, Ryu JK, Yoon D, Fearon ER, Rowe RG, Lee S, Maher CA, Weiss SJ, Yook Jl: A p53/miRNA-34 axis regulates Snail1-dependent cancer cell epithelial-mesenchymal transition. J Cell Biol 2011, 195(3):417-433.

41. Yu F, Yao H, Zhu P, Zhang X, Pan Q, Gong C, Huang Y, Hu X, Su F, Lieberman J, Song E: let-7 regulates self renewal and tumorigenicity of breast cancer cells. Cell 2007, 131(6):1109-1123.

42. Zhang Y, Yan LX, Wu QN, Du ZM, Chen J, Liao DZ, Huang MY, Hou JH, Wu QL, Zeng MS, Huang WL, Zeng YX, Shao JY: miR-125b is methylated and functions as a tumor suppressor by regulating the ETS1 proto-oncogene in human invasive breast cancer. Cancer Res 2011, 71(10):3552-3562.

43. Jansen MP, Reijm EA, Sieuwerts AM, Ruigrok-Ritstier K, Look MP, RodriguezGonzalez FG, Heine AA, Martens JW, Sleijfer S, Foekens JA, Berns EM: High miR-26a and low CDC2 levels associate with decreased EZH2 expression and with favorable outcome on tamoxifen in metastatic breast cancer. Breast Cancer Res Treat 2012, 133(3):937-947.

44. Schwarzenbach H, Milde-Langosch K, Steinbach B, Muller V, Pantel K: Diagnostic potential of PTEN-targeting miR-214 in the blood of breast cancer patients. Breast Cancer Res Treat 2012, 134(3):933-941.

45. Kuo TY, Hsi E, Yang IP, Tsai PC, Wang JY, Juo SH: Computational analysis of mRNA expression profiles identifies microRNA-29a/c as predictor of colorectal cancer early recurrence. PLoS One 2012, 7(2):e31587.

46. Stamatopoulos B, Meuleman N, Haibe-Kains B, Saussoy P, Van Den Neste E, Michaux L, Heimann P, Martiat P, Bron D, Lagneaux L: microRNA-29c and microRNA-223 down-regulation has in vivo significance in chronic lymphocytic leukemia and improves disease risk stratification. Blood 2009, 113(21):5237-5245.

47. Boll K, Reiche K, Kasack K, Morbt N, Kretzschmar AK, Tomm JM, Verhaegh G, Schalken J, von Bergen M, Horn F, Hackermuller J: MiR-130a, miR-203 and miR-205 jointly repress key oncogenic pathways and are downregulated in prostate carcinoma. Oncogene 2013, 32(3):277-285.

48. Li D, Zhao Y, Liu C, Chen X, Qi Y, Jiang Y, Zou C, Zhang X, Liu S, Wang X, Zhao D, Sun Q, Zeng Z, Dress A, Lin MC, Kung HF, Rui H, Liu LZ, Mao F, Jiang BH, Lai L: Analysis of MiR-195 and MiR-497 expression, regulation and role in breast cancer. Clin Cancer Res 2011, 17(7):1722-1730.

49. Buffa FM, Camps C, Winchester L, Snell CE, Gee HE, Sheldon H, Taylor M, Harris AL, Ragoussis J: microRNA-associated progression pathways and 
potential therapeutic targets identified by integrated mRNA and microRNA expression profiling in breast cancer. Cancer Res 2011, 71(17):5635-5645.

50. Ma L, Teruya-Feldstein J, Weinberg RA: Tumour invasion and metastasis initiated by microRNA-10b in breast cancer. Nature 2007, 449(7163):682-688.

51. King TD, Suto MJ, Li Y: The Wnt/beta-catenin signaling pathway: a potential therapeutic target in the treatment of triple negative breast cancer. J Cell Biochem 2012, 113(1):13-18.

52. Rakha EA, Chan S: Metastatic triple-negative breast cancer. Clin Oncol 2011, 23(9):587-600.

53. Shah SP, Roth A, Goya R, Oloumi A, Ha G, Zhao Y, Turashvili G, Ding J, Tse K, Haffari G, Bashashati A, Prentice LM, Khattra J, Burleigh A, Yap D, Bernard V, McPherson A, Shumansky K, Crisan A, Giuliany R, Heravi-Moussavi A, Rosner J, Lai D, Birol I, Varhol R, Tam A, Dhalla N, Zeng T, Ma K, Chan SK, et al: The clonal and mutational evolution spectrum of primary triple-negative breast cancers. Nature 2012, 486(7403):395-399.

doi:10.1186/1471-2407-14-51

Cite this article as: Avery-Kiejda et al:: Decreased expression of key tumour suppressor microRNAs is associated with lymph node metastases in triple negative breast cancer. BMC Cancer 2014 14:51.

\section{Submit your next manuscript to BioMed Central and take full advantage of:}

- Convenient online submission

- Thorough peer review

- No space constraints or color figure charges

- Immediate publication on acceptance

- Inclusion in PubMed, CAS, Scopus and Google Scholar

- Research which is freely available for redistribution 